\title{
Spatial Filtering with EViews and MATLAB
}

\author{
Robert Ferstl \\ Vienna University of Economics and Business Administration
}

\begin{abstract}
This article summarizes the ideas behind a few programs we developed for spatial data analysis in EViews and MATLAB. They allow the user to check for spatial autocorrelation using Moran's $\mathcal{I}$ and provide a spatial filtering procedure based on the $\mathcal{G}_{i}$ statistic by Getis and Ord (1992). We have also implemented graphical tools like Moran Scatterplots for the detection of outliers or local spatial clusters.
\end{abstract}

Zusammenfassung: Dieser Artikel beschreibt einige Programme, die wir zur Analyse räumlicher Daten in EViews und MATLAB entwickelt habe. Sie erlauben dem Anwender eine Überprüfung auf räumliche Autokorrelation mittels Moran's $\mathcal{I}$ und ermöglichen ein räumliches Filterungsverfahren basierend auf $\operatorname{der} \mathcal{G}_{i}$ Statistik von Getis and Ord (1992). Außerdem haben wir grafische Werkzeuge (z.B. Morans Streudiagramme) zur Analyse auf Ausreisser und zum Auffinden lokaler räumlicher Zusammenschlüsse implementiert.

Keywords: Spatial Autocorrelation, Moran Scatterplots, Spatial Filtering.

\section{Introduction}

In recent years spatial econometric methods gained in popularity. Nevertheless, the widely used econometric software package EViews ${ }^{1}$ does not contain functions for spatial data analysis. Therefore, we have developed a few scripts which can be started by a short entry in the command line. For users of the spatial econometrics toolbox by James P. LeSage ${ }^{2}$ the MATLAB ${ }^{3}$ version of the functions is an appropriate extension.

The programs calculate the global and local Moran's $\mathcal{I}$ statistic for spatial autocorrelation and its moments using the normal approximation and the more accurate saddlepoint approximation by Tiefelsdorf (2002). It is possible to check the data with graphical tools like Moran Scatterplots for outliers or local spatial clusters. Finally, we have implemented a filtering procedure based on the $\mathcal{G}_{i}$ statistic, which is another measure for local spatial dependence. So the user has an uncomplicated possibility to handle the presence of spatial autocorrelation in the data. For further details about the programs and the theoretical background see Ferstl (2004).

\footnotetext{
${ }^{1}$ http://www.eviews.com/

${ }^{2}$ http://www.spatial-econometrics.com/

${ }^{3}$ http://www.mathworks.com/
} 


\section{Spatial Structures}

\subsection{Global Spatial Structures}

The analysis of spatial data begins with a feasible measure to represent the inherent spatial dependence. The elements of a symmetric $n \times n$ spatial distance matrix $\boldsymbol{D}$ are defined by

$$
d_{i j}= \begin{cases}\sqrt{\left(\theta_{i}-\theta_{j}\right)^{2}+\left(\omega_{i}-\omega_{j}\right)^{2}}, & i \neq j \\ 0, & i=j\end{cases}
$$

where $(\theta, \omega)$ are the cartesian coordinates. In contrast to such a matrix, which measures dissimilarities, it is possible to use a spatial weight matrix to describe the similarities between the $i$-th and $j$-th spatial object.

There are several methods to transform a distance matrix to a global spatial weight matrix $\boldsymbol{G}$. We implemented the widely used negative exponential function to model the distance decay function (see e.g. Badinger et al., 2004). Therefore, the geographical distances are transformed to spatial weights by

$$
g_{i j}= \begin{cases}\exp \left(-\delta d_{i j}\right)>0 & \text { if objects } i \text { and } j \text { are spatially connected, } \\ 0 & \text { otherwise, }\end{cases}
$$

with $0<\delta<\infty$.

\subsection{Coding Schemes for Spatial Weight Matrices}

The programs provide three common coding schemes for standardization of the spatial weight matrix $\boldsymbol{G}$. Tiefelsdorf (2000) defines the linkage degree of the $i$-th spatial object by $d_{i}=\sum_{j=1}^{n} g_{i j}$. Therefore, the overall connectivity in $\boldsymbol{G}$ is $D \equiv \sum_{i=1}^{n} \sum_{j=1}^{n} g_{i j}$.

The globally standardized $C$-coding scheme is defined as

$$
\boldsymbol{C} \equiv \frac{n}{D} \boldsymbol{G} \text {. }
$$

In the row-sum standardized $W$-coding scheme the spatial weight matrix is transformed by

$$
\boldsymbol{W} \equiv[\operatorname{diag}(\boldsymbol{d})]^{-1} \boldsymbol{G},
$$

where $\boldsymbol{d}$ is a vector with the row-sums of $\boldsymbol{G}$. In the variance stabilizing $S$-coding scheme Tiefelsdorf (2000) defines the vector $\boldsymbol{q}$ by

$$
\boldsymbol{q} \equiv\left(\sqrt{\sum_{j=1}^{n} g_{1 j}^{2}}, \sqrt{\sum_{j=1}^{n} g_{2 j}^{2}}, \ldots, \sqrt{\sum_{j=1}^{n} g_{n j}^{2}}\right)^{\top} .
$$

As in the row-sum standardization the spatial weight matrix is transformed by $\boldsymbol{S}^{*} \equiv$ $[\operatorname{diag}(\boldsymbol{q})]^{-1} \boldsymbol{G}$. Finally, it is scaled by

$$
\boldsymbol{S} \equiv \frac{n}{Q} \boldsymbol{S}^{*}
$$

where $Q \equiv \sum_{i=1}^{n} \sum_{j=1}^{n} s_{i j}^{*}$.

Following the notation of Tiefelsdorf (2000), we use $\boldsymbol{V}$ as a placeholder for a spatial weight matrix which has been transformed by one of the three mentioned coding schemes. 


\subsection{Local Spatial Structures}

The local spatial weight matrix for the $i$-th spatial object consists of a copy of the $i$-th row and column of the global spatial weight matrix and zeros elsewhere. This definition leads to the following star-shaped form for the standardized local spatial weight matrices

$$
\boldsymbol{V}_{i} \equiv s_{i} \cdot\left(\begin{array}{ccccccc}
0 & \cdots & 0 & g_{1 i} & 0 & \cdots & 0 \\
\vdots & \ddots & \vdots & \vdots & \vdots & \ddots & \vdots \\
0 & \cdots & 0 & g_{i-1, i} & 0 & \cdots & 0 \\
g_{i 1} & \cdots & g_{i, i-1} & 0 & g_{i, i+1} & \cdots & g_{i n} \\
0 & \cdots & 0 & g_{i+1, i} & 0 & \cdots & 0 \\
\vdots & \ddots & \vdots & \vdots & \vdots & \ddots & \vdots \\
0 & \cdots & 0 & g_{n i} & 0 & \cdots & 0
\end{array}\right) .
$$

The scaling coefficient $s_{i}$ depends on the applied coding scheme (see Tiefelsdorf, 2000, p. 35)

$$
s_{i}=\left\{\begin{array}{cl}
n^{2} / 2 D, & \text { for the } C \text {-coding scheme, } \\
n / 2 d_{i}, & \text { for the } W \text {-coding scheme, } \\
n^{2} / 2 Q q_{i}, & \text { for the } S \text {-coding scheme. }
\end{array}\right.
$$

The sum over all local spatial weight matrices equals the global spatial weight matrix, i.e.

$$
\sum_{i=1}^{n} \boldsymbol{V}_{i}=\frac{1}{2}\left(\boldsymbol{V}+\boldsymbol{V}^{\top}\right) \text {. }
$$

\section{Spatial Autocorrelation}

\subsection{Global Moran's $\mathcal{I}$}

Having the spatial structure summarized in a spatial weight matrix, it is possible to define a measure for the spatial autocorrelation. The most prominent one is a statistic developed by Moran (1948), which is called Moran's $\mathcal{I}$ in the literature. According to Tiefelsdorf (2002) it is defined as follows.

Let $\boldsymbol{y}$ be a system of $n$ spatially distributed observations that are related to a set of $k$ exogenous variables via a linear regression model $\boldsymbol{y}=\boldsymbol{X} \boldsymbol{\beta}+\boldsymbol{\epsilon}$. The $n \times k$ design matrix includes the usual constant vector. The disturbances are distributed as $\boldsymbol{\epsilon} \sim \mathcal{N}\left(\mathbf{0}, \sigma^{2} \boldsymbol{\Omega}\right)$. The $n \times n$ matrix $\Omega$ reflects the covariance structure and $\sigma^{2}$ the variance of the disturbances. For independent disturbances the covariance structure is $\sigma^{2} \boldsymbol{I}$. The OLS regression residuals $\hat{\boldsymbol{\epsilon}}=\boldsymbol{M} \boldsymbol{y}$ are distributed as $\hat{\boldsymbol{\epsilon}} \sim \mathcal{N}\left(\mathbf{0}, \sigma^{2} \boldsymbol{M} \boldsymbol{\Omega} \boldsymbol{M}\right)$ using the projection matrix $\boldsymbol{M} \equiv \boldsymbol{I}-\boldsymbol{X}\left(\boldsymbol{X}^{\top} \boldsymbol{X}\right)^{-1} \boldsymbol{X}^{\top}$. For an underlying autoregressive spatial process $\boldsymbol{\Omega} \equiv(\boldsymbol{I}-\rho \boldsymbol{V})^{-1}\left(\boldsymbol{I}-\rho \boldsymbol{V}^{T}\right)^{-1}$, where $\rho$ is the spatial autocorrelation coefficient.

Global Moran's $\mathcal{I}$ is defined as a scale invariant ratio of quadratic forms in the normally distributed vector of regression residuals $\hat{\boldsymbol{\epsilon}}$

$$
\mathcal{I}=\frac{\hat{\boldsymbol{\epsilon}}^{\top} \frac{1}{2}\left(\boldsymbol{V}+\boldsymbol{V}^{\top}\right) \hat{\boldsymbol{\epsilon}}}{\hat{\boldsymbol{\epsilon}}^{\top} \hat{\boldsymbol{\epsilon}}}
$$

The multiplication with $\left(\boldsymbol{V}+\boldsymbol{V}^{\top}\right) / 2$ in the numerator ensures symmetry of the standardized spatial weight matrix. 


\subsection{Local Moran's $\mathcal{I}$}

Frequently the spatial dependencies are not the same for the whole dataset. Therefore, it is advisable to calculate a local test statistic. Local Moran's $\mathcal{I}_{i}$ is defined by using the local spatial weight matrix introduced in Section (2.3), i.e.

$$
\mathcal{I}_{i}=\frac{\hat{\boldsymbol{\epsilon}}^{\top} \boldsymbol{V}_{i} \hat{\boldsymbol{\epsilon}}}{\hat{\boldsymbol{\epsilon}}^{\top} \hat{\boldsymbol{\epsilon}}}
$$

Due to the additivity property in (1) the global Moran's $\mathcal{I}$ can be expressed as sum over all local Moran's $\mathcal{I}_{i}$.

\subsection{Moments of Moran's $\mathcal{I}$}

The moments of the global and local Moran's $\mathcal{I}$ statistic are calculated under the assumption of spatial independence. The moments of $\mathcal{I}$ can be expressed in terms of the resequenced spectrum of eigenvalues $\left\{\lambda_{1}, \ldots, \lambda_{n-k}, 0, \ldots, 0\right\}$ of $\boldsymbol{K} \equiv \boldsymbol{M}\left(\boldsymbol{V}+\boldsymbol{V}^{\top}\right) \boldsymbol{M} / 2$ (see Tiefelsdorf, 2000, chapter 9).

Expected value $\mathrm{E}[\mathcal{I}]=\mu_{1}$, variance $\operatorname{var}[\mathcal{I}]=\mu_{2}$, skewness $\mu_{3} /\left(\mu_{2}\right)^{3 / 2}$ and kurtosis $\mu_{4} /\left(\mu_{2}\right)^{2}$ of Moran's $\mathcal{I}$ are given by

$$
\begin{aligned}
\mu_{1} & \equiv \frac{\sum_{i=1}^{n-k} \lambda_{i}}{n-k}=\bar{\lambda} \\
\mu_{2} & \equiv \frac{2 \sum_{i=1}^{n-k}\left(\lambda_{i}-\bar{\lambda}\right)^{2}}{(n-k)(n-k+2)} \\
\mu_{3} & \equiv \frac{8 \sum_{i=1}^{n-k}\left(\lambda_{i}-\bar{\lambda}\right)^{3}}{(n-k)(n-k+2)(n-k+4)} \\
\mu_{4} & \equiv \frac{48 \sum_{i=1}^{n-k}\left(\lambda_{i}-\bar{\lambda}\right)^{4}+12\left(\sum_{i=1}^{n-k}\left(\lambda_{i}-\bar{\lambda}\right)^{2}\right)^{2}}{(n-k)(n-k+2)(n-k+4)(n-k+6)} .
\end{aligned}
$$

\subsection{Distribution of Moran's $\mathcal{I}$}

The $z$-transformed observed Moran's $\mathcal{I}_{o}$ is asymptotically normal distributed, i.e.

$$
z\left(\mathcal{I}_{o}\right)=\frac{\mathcal{I}_{o}-\mathrm{E}[\mathcal{I}]}{\sqrt{\operatorname{var}[\mathcal{I}]}} \approx \mathcal{N}(0,1)
$$

Tiefelsdorf (2000) was the first who published the exact reference distribution of Moran's $\mathcal{I}$. Its evaluation is complicated and computationally expensive. He proposed a saddlepoint approximation which is fast and accurate. Our software implementation yields the same results as the SPSS macro on the authors homepage ${ }^{4}$. For the mathematical background of the saddlepoint approximation see e.g. Tiefelsdorf (2002) or Ferstl (2004).

\footnotetext{
${ }^{4}$ http://geog-www.sbs.ohio-state.edu/faculty/tiefelsdorf/GeoStat.htm
} 


\section{Moran Scatterplots}

Anselin (1996) describes the Moran scatterplot as a tool for exploratory spatial data analysis (ESDA). Using the definition of global Moran's $\mathcal{I}$ in (2), it is possible to interpret $\mathcal{I}$ as the coefficient in a regression of the spatially lagged variable $\left(\boldsymbol{V}+\boldsymbol{V}^{\top}\right) \hat{\boldsymbol{\epsilon}} / 2$ on $\hat{\boldsymbol{\epsilon}}$. In other words, the slope of the regression line equals global Moran's $\mathcal{I}$.

Moran scatterplots can be used to find local patterns of spatial association. They also provide an easy way to detect outliers. Additionally, our programs plot a smoothed curve through the datapoints (instead of the regression line), and line diagrams of the studentized residuals and the Cook's distances. It is also possible to generate a so called Moran scatterplot matrix, which helps to visualize spatial interactions between two or more variables (see Anselin et al., 2002)

\section{Spatial filtering}

After having successfully detected spatial autocorrelation in the data, the question is how to handle it. The simplest alternative is to spatially filter the data, and estimate the model with the usual OLS procedure.

\subsection{The $\mathcal{G}_{i}$ Statistic}

A spatial filter is based on a local statistic of spatial dependence. Contrary to the version developed by Getis and Ord (1992), we use a non-binary weight matrix like in Section (2.1). This extension was introduced by Getis and Ord (1995) and was recently used in Badinger et al. (2004)

$$
\mathcal{G}_{i}(\delta)=\frac{\sum_{j=1}^{n} v_{i j}(\delta) x_{j}}{\sum_{j=1}^{n} x_{j}}, \quad j \neq i
$$

The $v_{i j}$ terms are the elements of a standardized weight matrix $\boldsymbol{V}$. The $x_{j}$ represent observations of a random variable $X_{j}$. The expected value of the $\mathcal{G}_{i}$ statistic is given by

$$
\mathrm{E}\left[\mathcal{G}_{i}\right]=\frac{V_{i}}{(n-1)}
$$

with $V_{i}=\sum_{j \neq i} v_{i j}(\delta)$. The variance is

$$
\operatorname{var}\left[\mathcal{G}_{i}\right]=\frac{V_{i}\left(n-1-V_{i}\right)}{(n-1)^{2}(n-2)} \frac{Y_{i 2}}{Y_{i 1}^{2}}
$$

where $\sum_{j=1}^{n} x_{j} /(n-1)=Y_{i 1}$ and $\sum_{j=1}^{n} x_{j}^{2} /(n-1)-Y_{i 1}^{2}=Y_{i 2}$.

The distribution of the $z$-standardized statistic $z\left(\mathcal{G}_{i}\right)$ is like Moran's $\mathcal{I}$ asymptotically $\mathcal{N}(0,1)$. The $\mathcal{G}_{i}^{*}$ statistic differs from the $\mathcal{G}_{i}$ statistic by including the value of $x_{i}$ if $i=j$. 


\subsection{The Filtering Process}

The expected value in (3) represents the realized value in the $i$-th region when no autocorrelation occurs. Dividing it by the $\mathcal{G}_{i}$ statistic results in a ratio that represents the spatially uncorrelated part of the data, i.e. the filtered variable $X^{*}$

$$
x_{i}^{*}=\frac{x_{i}\left(V_{i} /(n-1)\right)}{\mathcal{G}_{i}(\delta)} .
$$

Purely the spatial effects are stored in a new variable $L=X-X^{*}$. The goal is to minimize the remaining spatial autocorrelation in the filtered variable by varying the distance decay parameter $\delta$. That leads to the optimization of the objective function

$$
\delta_{\text {opt }}=\min _{\delta}\left|z_{\mathcal{I}}\left(\mathbf{X}^{*}\right)\right|, \quad 0<\delta<\infty
$$

\section{Programs}

Table 1 shows a list of available program files for EViews and MATLAB. Detailed descriptions of the syntax are included as comments. Demos of the functions can be found in the example files mentioned in Table 2.

\begin{tabular}{|c|c|c|}
\hline $\begin{array}{l}\text { EViews } \\
\text { subroutines }\end{array}$ & $\begin{array}{l}\text { MATLAB } \\
\text { functions }\end{array}$ & Description \\
\hline moransad.prg & moransad.m & $\begin{array}{l}\text { Global and local Moran's } I \\
\text { (including saddlepoint approximation) }\end{array}$ \\
\hline moranplot.prg & $\begin{array}{l}\text { moranplot.m } \\
\text { moranplotmatrix.m }\end{array}$ & $\begin{array}{l}\text { Moran scatterplot (with outlier detection) } \\
\text { Moran scatterplot matrix }\end{array}$ \\
\hline opt_delta.prg & & optimum delta for weights matrix \\
\hline $\begin{array}{l}\text { distance2weight.prg } \\
\text { getis.prg }\end{array}$ & $\begin{array}{l}\text { distance2weight.m } \\
\text { getis.m }\end{array}$ & $\begin{array}{l}\text { convert distance matrix to weight matrix } \\
\text { spatial filtering with Getis statistic }\end{array}$ \\
\hline optim.prg & objfct.m & one-dimensional optimization \\
\hline
\end{tabular}

Table 1: Programs

Table 2: Examples

\begin{tabular}{lll}
\hline $\begin{array}{l}\text { EViews } \\
\text { subroutines }\end{array}$ & $\begin{array}{l}\text { MATLAB } \\
\text { functions }\end{array}$ & Description \\
\hline ex_a.prg & ex_a.m & Example A: calculate Moran's $I$ \\
ex_b.prg & ex_b.m & Example B: Moran scatterplot for one variable \\
ex_c.prg & ex_c.m & Example C: Moran scatterplot for one variable (with outliers) \\
ex_d.prg & ex_d.m & Example D: Moran scatterplot matrix for two variables \\
ex_e.prg & ex_e.m & Example E: calculate optimum $\delta$ for spatial filtering \\
ex_f.prg & ex_f.m & Example F: spatial filtering with a given $\delta$ \\
\hline
\end{tabular}




\section{Practical Example}

The following examples are taken from Ferstl (2004). The analyzed series represents the gross value added per capita in million ECU in 1975 based on price and exchange rate levels from 1990. The $i=1, \ldots, 194$ spatial objects are NUTS 2 regions of the EU-15 countries. Table 3 shows the result of a test for global spatial autocorrelation. To perform the calculations in EViews these commands must be entered in the command line.

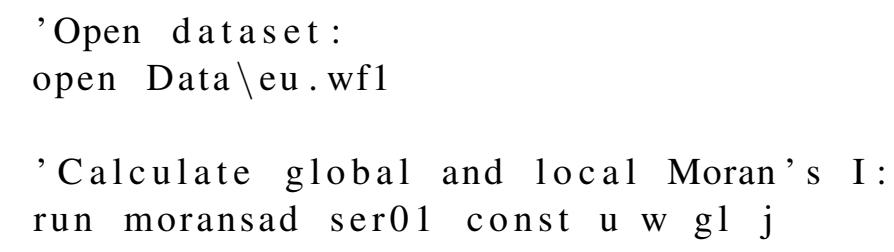

Table 3: Global Moran's $\mathcal{I}$ with normal and saddlepoint approximation

\begin{tabular}{|c|c|}
\hline \multicolumn{2}{|c|}{ Results using normal approximation } \\
\hline 0.6185 & Global Moran’s $\mathcal{I}$ \\
\hline-0.0052 & Expected Value \\
\hline 0.0006 & Variance \\
\hline 0.4355 & Skewness \\
\hline 3.3800 & Kurtosis \\
\hline 1.0000 & Probability $\left(\mathcal{I} \leq \mathcal{I}_{o}\right)$ \\
\hline 25.7052 & $z\left(\mathcal{I}_{o}\right)$ \\
\hline \multicolumn{2}{|c|}{ Results using saddlepoint approximation } \\
\hline 1.2877 & Saddlepoint \\
\hline 13.0177 & r-parameter for distribution \\
\hline 68.1026 & u-parameter for distribution \\
\hline 1.0000 & Probability $\left(\mathcal{I} \leq \mathcal{I}_{o}\right)$ \\
\hline
\end{tabular}

The analyzed variable shows a highly positive and significant spatial autocorrelation. Taking a look at a Moran scatterplot yields the same conclusion. Figure 1 also includes measures to identify influential observations. The following code constructs a Moran scatterplot in EViews.

'Moran scatterplot:

run moranplot ser01 w

The Moran scatterplot in Figure 1 shows a cluster of small values in the third quadrant. Alternatively, this local spatial dependence can be visualized by a choroplethe map of the $\mathcal{G}_{i}^{*}$ statistics, see Figure 2. There occur negative significant values in the region around Portugal.

The spatial filtering program finds a $\delta^{o p t}$ at 138.1611 . Figure 3 shows a plot of the objective function. Using the optimum $\delta$ parameter the spatial autocorrelation is successfully removed from the variable. The following commands are necessary to do this in EViews. 

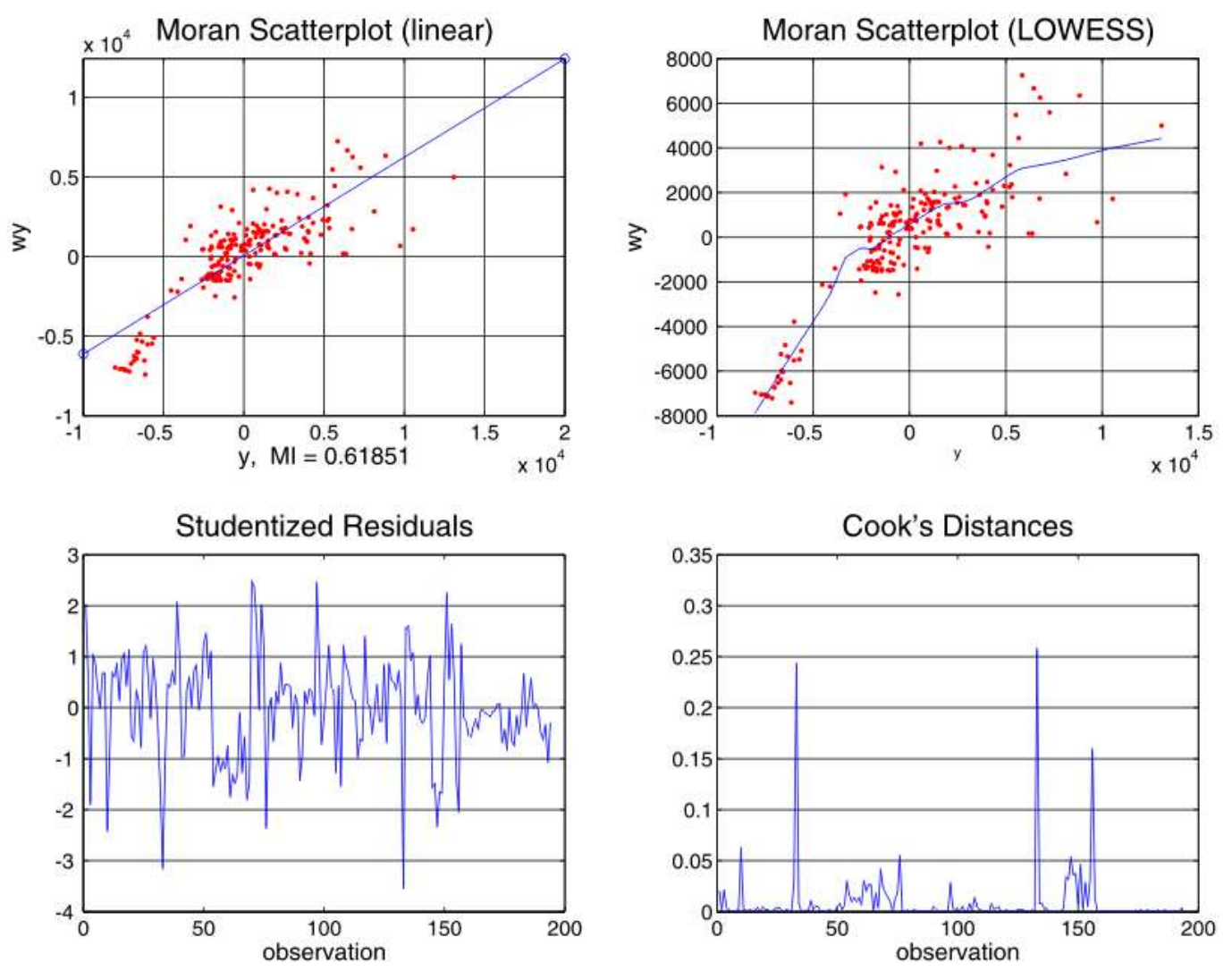

Figure 1: Moran Scatterplot with statistics for outlier detection

'Spatial filtering:

$\begin{array}{llllllllll}\text { run optdelta } & \text { ser01 } & \text { dst } & 70 & 80 & 100 & 0.00001 & 100\end{array}$

\section{Conclusions}

In this paper we shortly demonstrate a collection of programs we have developed for spatial data analysis in Ferstl (2004). The idea was to offer users of the widespread econometric software package EViews simple functions to analyze data for spatial dependencies. We have implemented functions to calculate Moran's $\mathcal{I}$ and its central moments using the normal and the saddlepoint approximation. Influential observations and spatial clusters can be visualized by Moran scatterplots. One way to deal with the presence of spatial autocorrelation is to filter the data. For this purpose functions are available, too.

All programs are available on request by email from the authors. They have been tested under Windows 2000/XP in EViews 4/5 and MATLAB 6/7.

\section{Acknowledgments}

We would like to thank the Department for Economic Geography and Geoinformatics at the Vienna University of Economics and Business Administration for providing us the 
Figure 2: Classified choroplethe map of the $\mathcal{G}_{i}^{*}$ statistics

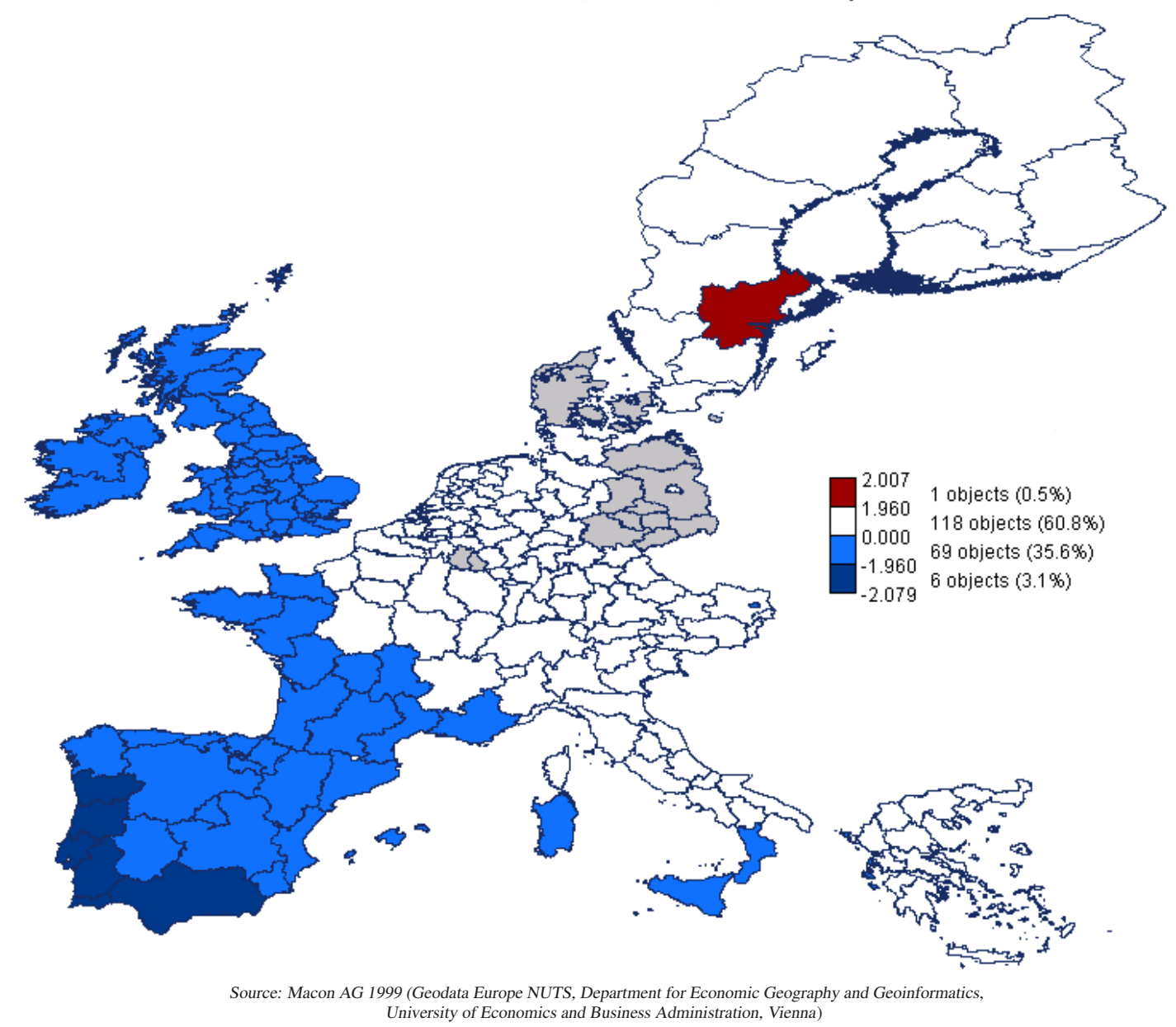

shape file used in the cartographic visualization.

\section{References}

Anselin, L. (1996). The Moran scatterplot as an ESDA tool to assess local instability in spatial association. In M. M. Fischer, H. J. Scholten, and D. Unwin (Eds.), Spatial Analytical Perspectives on GIS (p. 111-125). London: Taylor \& Francis Ltd.

Anselin, L., Syabri, I., and Smirnov, O. (2002). Visualizing Multivariate Spatial Correlation with Dynamically Linked Windows. (URL: http://agec221.agecon.uiuc.edu/ users/anselin/papers/multi_lisa.pdf)

Badinger, H., Müller, W., and Tondl, G. (2004). Regional convergence in the European Union, 1985-1999: A spatial dynamic panel analysis. Regional Studies, 38, 241253.

Ferstl, R. (2004). Werkzeuge zur Analyse räumlicher Daten - Eine Softwareimplementation in EViews und MATLAB. Unpublished master's thesis, Wirtschaftsuniversität Wien. 
Figure 3: Objective function for spatial filtering

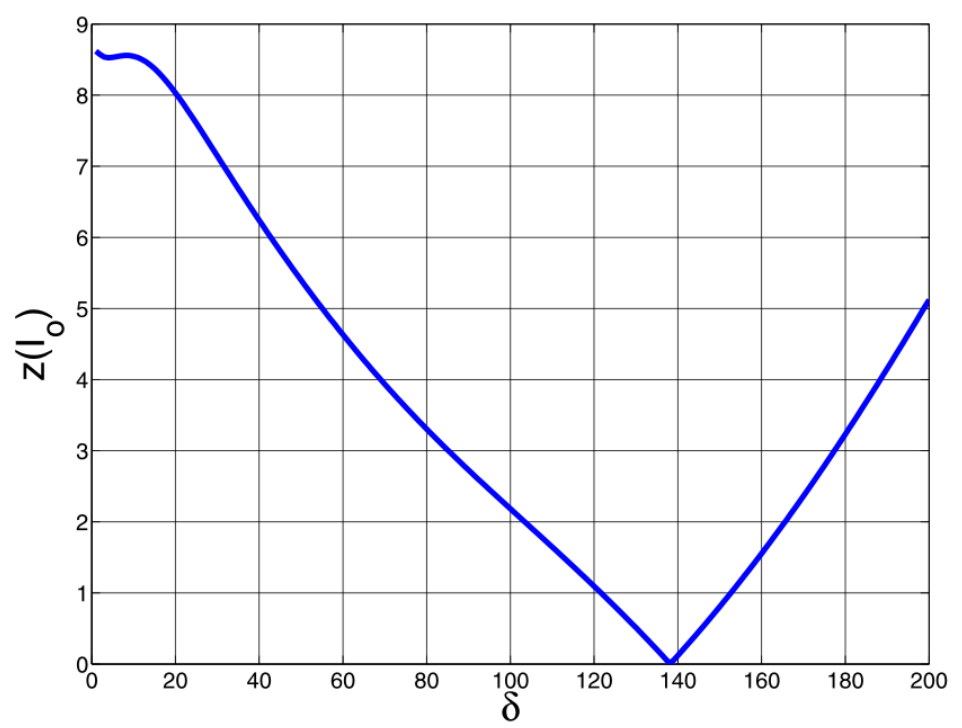

Getis, A., and Ord, J. K. (1992). The analysis of spatial association by use of distance statistics. Journal of Geographical Analysis, 24, 189-206.

Getis, A., and Ord, J. K. (1995). Local spatial autocorrelation statistics: Distributional issues and an application. Journal of Geographical Analysis, 27, 286-305.

Moran, P. A. P. (1948). The interpretation of statistical maps. Journal of the Royal Statistical Society, Series B, 10, 243-51.

Tiefelsdorf, M. (2000). Modelling Spatial Processes - The Identification and Analysis of Spatial Relationships in Regression Residuals by Means of Moran's I. Springer.

Tiefelsdorf, M. (2002). The saddlepoint approximation of Moran's $i$ 's and local Moran's $i_{i}$ 's reference distribution and their numerical evaluation. Journal of Geographical Analysis, 34, 187-206.

Author's address:

Robert Ferstl

Institute for Quantitative Management and Operations Research

Vienna University of Economics and Business Administration

Nordbergstraße 15

A-1090 Vienna, Austria

Tel. +43131336/ 4560

Fax $+43131336 / 708$

E-mail: robert.ferstl@wu-wien.ac.at

http://www.wu-wien.ac.at/or 